\title{
Discrepancy and nondiscrepancy methods of assessing visual-auditory interaction
}

\author{
DAVID H. WARREN and TIMOTHY J. McCARTHY \\ University of California, Riverside, California \\ and
}

ROBERT B. WELCH

University of Kansas, Lawrence, Kansas

\begin{abstract}
Much of our information about interaction among the sensory modalities has come from studies in which discrepancy between the modalities has been imposed experimentally. The question arises whether the conclusions generated from discrepancy research are affected by the use of the nonnatural experimental situation: Would the same conclusions be reached if discrepancy were not used? In addition to using the index of interaction derived from discrepancy, as in prior research, the present study assessed two nonexperimentally imposed indices to explore the interaction between vision and audition in a spatial localization task. The additional indices, or "tags," were standard deviations (SDs) and confidence ratings (CRs) of localization responses. The SD tag showed a pattern of interaction between vision and audition that was identical to that found with spatial discrepancy. The CR tag produced a similar pattern but did not show the same regular variation with the independent variable. Both SD and CR tags showed identical patterns of results when an experimentally imposed discrepancy was and was not used, suggesting that the use of moderate degrees of experimental discrepancy does not produce misleading conclusions about intersensory interaction.
\end{abstract}

If only one sensory modality receives information about an event, perception of that event is generally unambiguous. In many situations, however, information about an event is received by two (or more) modalities, and a question arises about the nature of the interaction between those modalities in the perception of such an event. Experimental situations may be designed to allow only one, or more than one, modality to receive information, and these situations can be used to study questions of intersensory interaction. We conclude that intersensory interaction has not occurred if the addition of a second sensory modality does not change the nature of the perception by the first modality. Often, however, the perception does change when information is available to a second modality, and in such a case we say that intersensory interaction has taken place. (When extreme influence of one modality on another occurs, the term "dominance" is sometimes used. "Interaction," however, is a term that seems to apply better to the general situation.)

A common way of arranging this experimental situation has been to place two modalities into conflict with one another, and then to examine the

This research was supported by a University of California Intramural Research grant to the first author. Send requests for reprints to David H. Warren, Department of Psychology, University of California, Riverside, California 92521. nature of the subject's perceptual resolution of the discrepancy. For example, Gibson (1933) had the subject look at his (the subject's) finger moving along a straight surface that was made to appear curved by means of a wedge prism; Young (1928) artificially transposed the information coming to the two ears, setting auditory and visual information into conflict; Hay, Pick, and Ikeda (1965) had the subject view his forefinger through a wedge prism, introducing a conflict between visual and proprioceptive information about the location of the finger. In the past decade and a half, numerous other investigators have used the "discrepancy" technique extensively to explore questions about the interactions of the senses.

The question arises whether the use of experimentally imposed discrepancy, a nonnatural stimulus situation, affects the nature of the conclusions that are drawn about intersensory interaction. Would the same conclusions about the nature of intersensory interaction be reached if conflict were not used? A primary objective of the present research was an examination of this issue.

The perception of spatial location by vision and audition was chosen for the research, as was the case in an earlier study by Warren, Welch, and McCarthy (1981). Using a 10-deg imposed spatial discrepancy, Warren et al. found the typical visual dominance effects: Visual localization was only 
weakly influenced by the addition of discrepant auditory information, but auditory localization was heavily influenced by the addition of discrepant visual information. These results were found when the stimulus situation was designed to induce a strong unity assumption, an assumption that the visual and auditory signals represented a single unitary event. This was done by having the subject view and hear a human reading a textual passage. (In the terminology of Welch \& Warren, 1980, the perceptual situation was one of high compellingness, in which compellingness referred to the relationship between the sensory modalities, not to the assumed veridicality or reliability of one sensory modality as compared with another.) In contrast, when the stimulus situation was reduced in compellingness, Warren et al. found significantly less visual influence on audition, and no auditory influence on vision. Whereas the subject in the high-compellingness situation responded as if the discrepant visual and auditory signals signified a single location, the subject in the low-compellingness situation responded as if the two modalities were delivering information about separate spatial events.

Warren et al. (1981) concluded that when stimulus conditions are such as to induce a strong assumption of unity (that both modalities are giving information about a single event), the subject relies heavily on visual and much less heavily, although still significantly, on auditory information in his or her decision about the location of the event. With a decrease in the compellingness of the stimulus situation, the degree of interaction between the two modalities decreases significantly, but with the primary reliance still on the visual information.

The question now at issue is whether the same conclusions about the interaction of vision and audition in the perception of spatial location would be drawn if spatial discrepancy were not involved in the experimental setting. In fact, Welch and Warren (1980), in their review of the discrepancy literature, suggested that an imposed discrepancy, the very crux of the experimental procedure, may interfere with the normal manner of intersensory interaction by lessening the subject's inclination to assume that the information from the two senses represents a unified, single event.

Indeed, the traditional measure of the extent to which one modality is influenced by another depends upon the use of discrepancy. It is by means of imposed discrepancy that the information from the two modalities can be made separately identifiable, so that the experimenter can keep track of which modality is influencing which, and how strongly. The experimental problem posed by this dilemma can be resolved only by finding a different way of keeping track of the contributions of the two modalities, of "tagging" the information from the two modal- ities, as it were, so that their relative contributions to the bisensory percept can be traced and identified. Any such alternative index, or "tag," of a modality's information must have different values for the two modalities in question; otherwise, it would not serve to distinguish their contributions to a bisensory percept.

A good candidate for such an index is trial-totrial variability. Humans localize visual targets much less variably (i.e., more precisely) than they do auditory targets. The procedure, then, would be to assess, for each subject, variability of localization of visual targets and of auditory targets separately, and then to examine the variability of localization of targets about which the subject receives both visual and auditory information simultaneously. Comparison of the bisensory variability with vision-alone and audition-alone variabilities should show the relative roles of the visual and the auditory information in the bisensory percept. The logic is identical to the use of spatial-location discrepancy as a tag or modality index, but it is important to note that variability can be measured in the absence of spatial discrepancy, and thus provides a way of comparing conclusions about intersensory relations when spatial discrepancy is and is not imposed.

Another aspect of spatial localization that may differentiate visual and auditory performance, and therefore serve as a useful index of the relative contributions of the modalities, is the subjects' confidence in the accuracy of their judgments: Subjects report more confidence in their judgments of visual than of auditory targets. In the present research, confidence ratings were recorded for localization judgments, using a 7-point scale. Thus, three indices of the contributions of the modalities were available-mean localization judgment, trial-to-trial variability of localization judgments, and confidence in localization judgments.

Briefly, the experimental design called for four groups of subjects to be tested in the following conditions: (1) 10-deg discrepancy between visual and auditory locations and a highly compelling visualauditory situation, (2) no discrepancy and a highly compelling situation, (3) 10-deg discrepancy and a low-compellingness situation, and (4) no discrepancy and a low-compellingness situation. In each condition, each subject made magnitude estimation judgments in four localization tasks-visual-only target information, auditory-only target information, visual + auditory target information with visual localization requested, and visual + auditory target information with auditory localization requested. For each task, mean localization judgment, variability of localization responses, and mean confidence rating of localization responses were calculated, thus generating three indices of the relative contributions of vision and audition to bisensory localization per- 
ceptions, and thus potentially three sets of conclusions about the nature of intersensory organization. The two primary research questions were (1) whether these sets of conclusions would differ from one another, and (2) whether the conclusions drawn would differ depending upon the presence or absence of spatial discrepancy.

As noted, the four conditions were formed by the combination of the two independent variables, discrepancy vs. no discrepancy and high vs. low compellingness. On the basis of the Welch and Warren review (1980) and the results of Warren et al. (1981), the predictions for these several conditions were as follows. Decreasing compellingness decreases the unity assumption (UA) and thus should decrease the subject's tendency to regard the visual and auditory information as signaling a single event. Imposing a location discrepancy should also lessen the UA. Therefore, the high-compellingness/no-discrepancy condition should produce the strongest UA and thus the greatest degree of intersensory interaction, and the low-compellingness/discrepancy condition should produce the least. The remaining two conditions should show intermediate amounts of interaction.

\section{METHOD}

\section{Subjects}

A total of 48 male and female college students with normal or corrected vision served as subjects and received either pay or course-related credit. Each group of 12 received one of the four experimental conditions.

\section{Apparatus}

The subject sat at a semicircular shelf with head fixed in a combination chinrest and forehead restraint. The subject viewed the visible targets monocularly with the right eye through a clear Plexiglas window with a $60-\mathrm{deg}$ field of view. A shutter prevented vision except when it was involved in the task. Beyond the perimeter of the shelf was another shelf holding a 9-in. TV monitor, which could be placed at a variety of target positions. The audio signal was fed through a separate speaker, which could also be positioned at various target locations; it was hidden behind a cloth fence and not visible to the subject. Thus, it was possible to give the subject an auditory-only target (for which the eyepiece shutter remained closed), a visual-only target (with the speaker not activated), or a bisensory target (visual and auditory information) with or without a 10-deg discrepancy introduced. The discrepancy approximated the optical effect of a 20diopter base-left wedge prism. The target locations were at 0 , $\pm 5, \pm 10, \pm 15$, and \pm 20 deg relative to the subject's straight ahead.

The videoscreen was the only source of illumination during the experimental sessions. The walls of the room and the shelf in front of the subject were covered with foam rubber to deaden echoes.

\section{Procedure}

A magnitude estimation (ME) procedure was used for all localization responses. The subject was instructed to use the number zero to denote the location straight ahead of his or her nose, positive numbers for locations to his or her right, and negative numbers for locations to the left. The subject was shown the visual target in the +20 - and -20 -deg locations and was requested to use the largest numbers, positive and negative, for these extreme locations.
A second response was a confidence rating (CR). The subject was instructed to use the letters $A$ through $G$ to indicate degree of confidence in the accuracy of each ME location response. The subject first gave a ME of the location of the target and then selected the letter that best indicated his or her confidence in the accuracy of that response.

A training procedure was used in which the subject was presented with visual-only and auditory-only targets across the entire 40-deg range and was asked for judgments of location and CR. Ordinarily, 15 practice trials ensured that the subject understood the procedure and was using the scales with good reliability.

Each subject participated in four localization tasks. The first two were control tasks, one with visual targets alone (called $\mathrm{Vc}$, for visual control) and the other with auditory targets alone (called Ac, for auditory control). For each task, the targets were presented at each of the nine target locations twice in random order, for a total of 18 trials per task. Half the subjects in each group performed $V c$ first, and the other half, Ac first.

The second pair of tasks involved presentation of visual and auditory targets simultaneously and are referred to as the bisensory tasks. In both, the shutter was lifted at the same time that the speaker was activated, so that the subject received the two signals simultaneously. In one of these tasks, called Ve (for visual exposure), the subject was asked to make a target localization based only on the visual information, and in the other, called Ae (for auditory exposure), he or she was asked to localize based only on the auditory information. In each task, the subject made two judgments of each target; the targets are described below. Half of the subjects performed the Ve task first, and the other half, the Ae task first. (Following Pick, Warren, \& Hay, 1969, the bisensory tasks in the conflict paradigm are typically presented after the control tasks in order to prevent the possibility of any adaptation occurring as a result of the bisensory tasks, thereby affecting the control task values.)

After the four localization tasks, two supplementary measures were obtained. The first was an assessment of the subject's unity assumption (UA). The subject made one UA response, using a 7-point scale with 7 denoting a high degree of assumed unity between the visual and auditory signals in the bisensory tasks and 1 denoting a low degree of assumed unity. The second supplementary measure was an assessment of perceived discrepancy (PD). The subject made a single judgment, using his or her ME scale for location, of the perceived spatial separation between the visual and auditory signals in the bisensory tasks.

In the bisensory tasks, the subjects in the discrepancy condition had the auditory signal $10 \mathrm{deg}$ to their left of the visual signal. Thus, there were seven bisensory target combinations, in which the visual target varied in 5-deg steps from -10 to +20 deg and the corresponding auditory target varied from -20 to $+10 \mathrm{deg}$. More extreme rightward and leftward target pairs were not used because one of the members of the pair would have fallen outside the designated -20 - to +20 -deg range. In the no-discrepancy condition, the signals were spatially coincident, and all nine targets, ranging in 5-deg steps from -20 to $+20 \mathrm{deg}$, were used. Half of the subjects were in the high-compellingness condition, in which the face of a male human speaker appeared on the videoscreen while his voice read a passage from a text; the other half were in the low-compellingness condition, in which the auditory signal was again the male voice. The videoscreen was blank, with only a piece of tape as the visual target at the location where the speaker's mouth would have been.

\section{RESULTS}

Broadly stated, the purpose of the various analyses was to examine two major questions about intersensory interaction and the several ways of assessing it. First, in conditions with imposed location discrepancy, are the conclusions about intersensory in- 
teraction drawn from the location response the same as those obtained from the other concurrently obtained responses, confidence rating (CR) and standard deviation (SD), which are not created by discrepancy? Second, do responses (SD and CR) that are not dependent on discrepancy produce the same conclusions about intersensory interaction in conditions not involving experimentally imposed discrepancy as they do in conditions with discrepancy?

\section{Data Reduction}

Because the subjects in the discrepancy and nodiscrepancy conditions received different target combinations in the bisensory tasks, different target ranges were involved in the calculation of the various summary measures. The subjects in the nodiscrepancy condition judged two targets at each of the nine target locations in each of the control tasks and in each of the bisensory tasks; thus, data for 18 targets were used for each of the four tasks. The subjects in the discrepancy condition judged only 14 targets in each of the bisensory tasks, as noted earlier: For the Ae task, the auditory targets ranged from -20 to $+10 \mathrm{deg}$, and for the Ve task, the visual targets ranged from -10 to $+20 \mathrm{deg}$. The appropriate control for the Ve targets therefore consists of the $\mathrm{Vc}$ targets in the -10 - to +20-deg range, and the appropriate control for the Ae targets consists of the Ac targets in the -20 - to +10-deg range. Thus, only these $14 \mathrm{Ac}$ and $14 \mathrm{Vc}$ trials were used to produce the summary measures. (All 18 trials were actually judged by the subject in each of the Ac and Vc tasks, in order to avoid creating a response bias that might carry over into the bisensory tasks, but only the 14 relevant trials were used in the calculations of the summary measures for Ac and $\mathrm{Vc}$.)

Before describing the transformation of ME judgments to degree values, it should be noted that the relationship between target location and $\mathrm{ME}$ ratings was extremely strong and highly linear. Pearson product-moment correlations were calculated for each task for each subject and ranged from .83 to 1.00 , with only 4 of the 192 (4 groups $\times 12$ subjects $\times 4$ tasks) correlation coefficients falling below .90. Clearly, then, there was no unwanted interaction of target location and ME judgment.

The ME judgments were transformed to degree values, independently for each task for each subject, by the following procedure: (a) Sum the absolute values of the target locations in degrees, (b) sum the absolute values of the ME judgments, (c) divide (a) by (b) to obtain the conversion factor representing the ratio of degree units to ME units, and (d) multiply each ME judgment by the conversion factor to transform ME judgments to degrees. (The conversion factor was also applied to the PD judgments to produce PD scores in degrees.)
A mean localization score (mean transformed MEs) for each task was then generated for each subject on each task. Each such localization score, in degrees, was subtracted from the true value for that trial, yielding a distribution of error scores for each subject on each task. The SD of each such distribution was then calculated, generating a SD score for each subject on each task. Mean CR scores were also calculated for each task.

In order to represent the amount of intersensory influence, visual and auditory shift scores were calculated for localization, SD, and CR data. The auditory shift score for localization, for instance, was calculated by taking the difference between the auditory-alone control mean (Ac) and the mean of the bisensory task when auditory localization was requested (Ae). For the localization measure, signs were assigned to represent a shift toward the control value of the other modality ( $\mathrm{Vc}$ in this example) as positive. Say that, for a given subject, the mean transformed localization of the auditory target alone (Ac) is $0 \mathrm{deg}$ and the mean transformed localization of the visual target alone (Vc) is $10 \mathrm{deg}$. If on those bisensory trials when the subject is asked where the voice is heard to be coming from (Ae) the mean transformed response is $4 \mathrm{deg}$, then the auditory shift score for this subject is: $\mathbf{A e}-\mathbf{A c}$, or $4 \mathrm{deg}-0 \mathrm{deg}=$ +4 deg. The sign of the shift score is positive because the shift in auditory localization from Ac $(0 \mathrm{deg})$ to Ae $(4 \mathrm{deg})$ is in the direction of the Vc value (10 deg). For the SD and CR measures, positive scores were assigned to represent lower variability and higher confidence on the bisensory as compared with the control tasks. These shift scores were used in the analyses reported below. The $\alpha$ level was set at .05 for all effects.

\section{Localization}

Following transformation of the ME localization data and generation of localization shift scores, auditory- and visual-localization shift scores were entered into an analysis of variance with discrepancy (10 deg vs. no discrepancy) and compellingness (high vs. low) as between-subjects factors and modality (visual vs. auditory shift) as a within-subjects factor. Mean shift scores appear in Table 1. The main effects of compellingness $(F=14.02)$, discrepancy $(F=$ 33.04), and modality $(F=35.64)$ were all highly significant ( $\mathrm{df}=1,44 ; \mathrm{p}<.01$ ), indicating, as expected, more shift with high compellingness, more shift with 10-deg discrepancy, and more auditory than visual shift. However, two interactions also proved significant, that of compellingness $\times$ modality $[F(1,44)=$ $5.44, p<.05]$ and that of modality $\times$ discrepancy $[F(1,44)=16.93, p<.01]$. The remaining interactions were not significant. The compellingness $\times$ modality interaction is not particularly interesting. There was relatively little visual shift, so visual shift was 
Table 1

Mean Task Scores (With Standard Deviations in Parentheses) and Mean Shift Scores for Localization, SD, and CR (With 95\% Confidence Intervals in Parentheses)

\begin{tabular}{|c|c|c|c|c|c|c|}
\hline & $V_{c}$ & $\mathrm{Ac}$ & $\mathrm{Ve}$ & $\mathrm{Ae}$ & $A_{\text {shift }}$ & $\mathrm{V}_{\text {shift }}$ \\
\hline \multicolumn{7}{|c|}{ Localization: 10-Deg Discrepancy } \\
\hline $\begin{array}{l}\text { Hi Comp } \\
\text { Lo Comp }\end{array}$ & $\begin{array}{l}5.73(2.20) \\
5.64(1.90)\end{array}$ & $\begin{array}{l}-4.66(1.96) \\
-4.00(1.89)\end{array}$ & $\begin{array}{l}4.99(1.85) \\
5.49(2.46)\end{array}$ & $\begin{array}{r}2.14(3.97) \\
-1.02(4.11)\end{array}$ & $\begin{array}{l}6.80(4.13) \\
2.98(1.90)\end{array}$ & $\begin{array}{l}.74(.51) \\
.15(.80)\end{array}$ \\
\hline \multicolumn{7}{|c|}{ Localization: No Discrepancy } \\
\hline $\begin{array}{l}\text { Hi Comp } \\
\text { Lo Comp }\end{array}$ & $\begin{array}{r}2.79(2.48) \\
.01(1.03)\end{array}$ & $\begin{array}{l}.67(1.93) \\
.36(.87)\end{array}$ & $\begin{array}{r}2.61(4.80) \\
-.05(.52)\end{array}$ & $\begin{array}{r}2.10(2.05) \\
.05(.60)\end{array}$ & $\begin{array}{r}1.43(1.70) \\
.31(.37)\end{array}$ & $\begin{array}{r}.16(.81) \\
-.06(.26)\end{array}$ \\
\hline \multicolumn{7}{|c|}{ SD: 10-Deg Discrepancy } \\
\hline $\begin{array}{l}\text { Hi Comp } \\
\text { Lo Comp }\end{array}$ & $\begin{array}{l}3.47(.87) \\
2.66(.66)\end{array}$ & $\begin{array}{l}5.10(1.72) \\
4.04(1.58)\end{array}$ & $\begin{array}{l}3.12(1.11) \\
2.44(.82)\end{array}$ & $\begin{array}{l}3.62(1.20) \\
3.54(1.06)\end{array}$ & $\begin{array}{r}1.48(.96) \\
.50(.45)\end{array}$ & $\begin{array}{l}.36(.39) \\
.22(.40)\end{array}$ \\
\hline \multicolumn{7}{|c|}{ SD: No Discrepancy } \\
\hline $\begin{array}{l}\text { Hi Comp } \\
\text { Lo Comp }\end{array}$ & $\begin{array}{l}2.96(.91) \\
1.76(.32)\end{array}$ & $\begin{array}{l}4.33(1.06) \\
3.50(.90)\end{array}$ & $\begin{array}{l}2.66(1.15) \\
1.66(.32)\end{array}$ & $\begin{array}{l}2.93(1.83) \\
2.83(1.05)\end{array}$ & $\begin{array}{r}1.40(.94) \\
.67(.56)\end{array}$ & $\begin{array}{l}.30(.49) \\
.10(.22)\end{array}$ \\
\hline \multicolumn{7}{|c|}{ CR: 10-Deg Discrepancy } \\
\hline $\begin{array}{l}\text { Hi Comp } \\
\text { Lo Comp }\end{array}$ & $\begin{array}{l}2.53(.56) \\
2.59(.59)\end{array}$ & $\begin{array}{l}2.93(.85) \\
2.89(.79)\end{array}$ & $\begin{array}{l}2.31(.67) \\
2.41(.71)\end{array}$ & $\begin{array}{l}2.55(.70) \\
2.67(.74)\end{array}$ & $\begin{array}{l}.38(.34) \\
.22(.20)\end{array}$ & $\begin{array}{l}.22(.28) \\
.18(.25)\end{array}$ \\
\hline \multicolumn{7}{|c|}{ CR: No Discrepancy } \\
\hline $\begin{array}{l}\text { Hi Comp } \\
\text { Lo Comp }\end{array}$ & $\begin{array}{l}2.03(.57) \\
2.07(.50)\end{array}$ & $\begin{array}{l}2.72(.77) \\
2.67(.39)\end{array}$ & $\begin{array}{l}1.99(.56) \\
1.93(.35)\end{array}$ & $\begin{array}{l}2.37(.79) \\
2.36(.46)\end{array}$ & $\begin{array}{l}.35(.28) \\
.31(.31)\end{array}$ & $\begin{array}{l}.04(.16) \\
.14(.18)\end{array}$ \\
\hline
\end{tabular}

Note-Localization = localization ME scores converted to degrees; positive scores are in the direction of the other modality's control value. $S D=S D$ scores in degrees; positive scores represent improved (i.e., less variable) responding. $C R=C R$ scores on 7.point $C R$ scale; positive scores represent shifts in the direction of increased confidence.

not susceptible to the effect of compellingness: The simple main effect of compellingness for the visual modality was not significant $[F(1,44)=2.38$, n.s. $]$. There was substantial auditory shift, however, and the simple main effect of compellingness for audition was significant $[F(1,44)=14.44, p<.01]$, with high compellingness producing much more shift than low compellingness, as predicted. The interaction of modality with discrepancy was, of course, produced by the fact that in the no-discrepancy condition the auditory and visual targets were coincident and could produce no reliable shift: The simple main effect of modality under no discrepancy was not significant $[F(1,44)=3.96$, n.s. $]$. In the discrepancy condition, however, substantial shifts occurred, with the auditory shift occurring far more strongly than the visual shift $[F(1,44)=26.01, p<.01]$.

In essence, the results replicated those reported by Warren et al. (1981). There was more interaction between the two modalities when compellingness was high than when it was low; when spatial discrepancy was involved, the visual information was relied upon far more heavily than was the auditory information. It should again be emphasized that spatial location is not a useful "tag," or index, of the two modalities' contributions, when discrepancy is not imposed-the two modalities are being given the same information, and thus they cannot be separately identified in the bisensory percept.

\section{Standard Deviation}

As expected, the mean Vc SD was significantly lower than the mean Ac SD $[\mathrm{t}(47)=7.65, \mathrm{p}<.01]$.
The experimental question, however, lies in the relationship of the mean SD scores for the Ae and the Ve bisensory tasks. It was hypothesized that the presence of visual information during Ae performance would lower the mean Ae SD score, and would lower it more when compellingness was high than when it was low. It was not expected, however, that the presence of the (less precisely localizable) auditory information during Ve performance would worsen the mean $\mathrm{Ve} \mathrm{SD}$, since there is no reason to suppose that the subject should perform less well than he or she does when vision alone is available.

SD shift scores were entered into an analysis of variance (ANOVA) with compellingness and discrepancy as between-subjects factors and modality as a within-subjects factor. There was a significant effect of compellingness $[F(1,44)=9.93, p<.01]$, with more shift in high- than in low-compellingness conditions. There was a significant effect of modality $[F(1,44)=31.11, p<.01]$, showing far more auditory shift than visual shift. There was no effect of discrepancy, and no interaction with discrepancy was significant. The interaction of compellingness and modality was significant $[F(1,44)=6.21, p<.05]$. As in the case of the localization results, the simple main effect of compellingness on auditory shift was significant $[F(1,44)=16.11, p<.05]$, with greater shift under high compellingness. Visual shift was of such small magnitude that the simple main effect of compellingness was not significant $[F(1,44)<1.0]$.

These effects are straightforward and as hypothesized. The absence of any effect of discrepancy is notable because it demonstrates that the use of an 
experimentally imposed location discrepancy does not change the pattern of results: The same conclusions about intersensory interaction would be drawn from the 10-deg discrepancy conditions as from the no-discrepancy conditions. The modality effect was the same as for the localization performance, with vision influencing audition substantially and audition influencing vision very little. The compellingness effect also paralleled that found with localization: More intersensory influence occurred under high than under low compellingness.

Thus, the use of the naturally occurring SD index, an index that does not depend on an experimentally induced discrepancy, showed the same pattern of variation with the independent variables that the experimentally induced location discrepancy index did. It may be concluded that the pattern of intersensory interaction developed from discrepancy research is not idiosyncratic to the artificial discrepancy paradigm.

\section{Confidence Rating (CR)}

It was hypothesized that $C R$ would follow the same outcome as SD. The subjects should show less confidence in their Ac than in their Vc judgments, and, in fact, they did $[t(47)=5.00, p<.001]$. Furthermore, the subjects' confidence in their auditory judgments should improve when visual information accompanies the auditory, and more so in the highly compelling stimulus situation than in the lowcompelling situation. Confidence in visual judgments was not expected to worsen when the visual information was accompanied by auditory information.

The auditory and visual shift scores were entered into an ANOVA with compellingness, discrepancy, and modality factors. The outcome of the ANOVA supported the expectations only in part. A weak effect of modality did emerge $[F(1,44)=5.53, p<.05]$, with more auditory shift (about one-third of a category) than visual (about half that), as expected. As predicted, there was no effect of discrepancy. However, there was no main effect of compellingness, in contrast with the results for SD and localization judgments. No interactions were significant.

\section{Unity Assumption (UA) and Perceived \\ Discrepancy (PD)}

Welch and Warren (1980) reviewed the literature on intersensory interaction that makes use of the discrepancy paradigm, and proposed a model of interaction to account for the various findings. One aspect of their formulation is that the observer in a bisensory situation makes an assumption (the UA) about whether the information coming to the two modalities represents a single event. It was predicted, in the present study, that UA should be highest in the no-discrepancy/high-compellingness condition, lowest in the discrepancy/low-compellingness condi-
Table 2

Mean UA and PD Scores, With 95\% Confidence Intervals (CI)

\begin{tabular}{rcccccc}
\hline & & \multicolumn{2}{c}{ UA } & & \multicolumn{2}{c}{ PD } \\
\cline { 6 - 7 } \cline { 5 - 6 } $\begin{array}{c}\text { Discrep- } \\
\text { ancy* }\end{array}$ & $\begin{array}{c}\text { Compel- } \\
\text { lingness }\end{array}$ & Mean & CI & & Mean & CI \\
\hline 10 & High & 3.92 & 1.14 & & 2.00 & 1.97 \\
10 & Low & 2.67 & .98 & & 4.51 & 1.56 \\
0 & High & 4.92 & .62 & .33 & .51 \\
0 & Low & 3.00 & .42 & -.50 & .86 \\
\hline
\end{tabular}

*In degrees.

tion, and intermediate in the two remaining conditions. Using the rating of UA made by each subject, these predictions of the model were tested. The UA data were submitted to a two-way ANOVA with discrepancy and compellingness as factors: mean UA scores appear in Table 2 . The effect of compellingness was highly significant, showing UA to be greater with high compellingness than with low $[F(1,44)=$ $8.97, p<.01]$. The effect of discrepancy was not significant $(p>.05)$, however, indicating that discrepancies as small as $10 \mathrm{deg}$ do not weaken the UA. Presumably, if the imposed discrepancy were increased (as in Warren \& Cleaves, 1971, and Warren et al., 1981), UA would weaken significantly. The interaction of compellingness and discrepancy was not significant $(F<1.0)$.

The Welch and Warren (1980) model proposes that intersensory interaction in discrepancy situations serves the role of reducing perceived discrepancy so that unified perception can occur. To the extent that intersensory interaction is not complete, a PD should remain. The mean PD scores, in degrees, appear in Table 2. Significant PD should, of course, occur only in the discrepancy groups. Table 2 bears this out. Furthermore, PD should be greater for the lowcompellingness/discrepancy group than for the highcompellingness/discrepancy group. The PD scores were submitted to a $t$ test, and the difference between the two discrepancy groups was significant in the predicted direction $[\mathrm{t}(11)=1.88, \mathrm{p}<.05]$.

Finally, the Welch and Warren (1980) model predicts an inverse relationship between UA and PD. The Pearson correlation between these scores, for the combined discrepancy groups, was -.55 ( $\mathrm{p}<$ $.01)$.

\section{DISCUSSION}

As noted earlier, intersensory interaction is demonstrated if the perception of an event by one modality is changed when it is accompanied by another modality's receiving information about the event. The present study examined first whether the nature of intersensory interaction is changed by an artificially imposed discrepancy between the modalities, and second whether conclusions about the nature of intersensory interaction are different when alterna- 
tive indices are used that do not depend on the experimental discrepancy manipulation.

Both questions received definitive answers. First, does the use of an experimental discrepancy affect the pattern of results? Meaningful examination of this question is impossible using the localization index, since the two modalities receive identical location information in the no-discrepancy condition. The SD measure does test the question, and the pattern of conclusions was identical whether or not discrepancy was involved: There was no main effect of or interaction with the discrepancy factor. For both the discrepancy and the no-discrepancy conditions, visual information predominated in the bisensory percept, the more so when compellingness was high than when it was low. The same conclusion is drawn from the CR data: There was no effect of, or interaction with, discrepancy. These results should prove reassuring to those who use the discrepancy paradigm to study intersensory interaction. However, the reassurance should be taken with some caution, since it is logically apparent (Welch \& Warren, 1980) ánd has been demonstrated experimentally (Warren \& Cleaves, 1971; Warren et al., 1981) that when discrepancy is increased dramatically, the pattern of intersensory interaction does change significantly.

Second, do alternative indices to the location discrepancy measure show the same pattern of intersensory interaction? The answer in this case is a qualified yes. Analysis of the SD measure showed a virtually identical outcome: There was significantly more intersensory interaction under the highcompellingness condition, and visual information affected auditory far more than vice versa. The CR measure also showed a greater predominance of vision, although the expected effect of compellingness did not occur.

The CR measure was selected not, like the SD and localization measures, as a direct measure of performance, but rather as an index of a more metaperceptual kind. That is, it measures not how the subject performs, but how the subject thinks he or she performs. The data show that $\mathrm{CR}$ did not vary with the independent variables in the same way that the more directly perceptual SD and localization measures did. The degree of consistency in the CR data across the four groups is striking: Auditory shift ranged only between .22 and .38 , and visual shift ranged between .04 and .22 .

The lack of regular variation with the independent variables suggests that the CR measure may not have been reflective of perceptual processes at all. Instead, the data may reflect the subject's belief that he or she is better at visual localization than at auditory localization and thus should structure $C R$ responses accordingly. Alternatively, the subject may have been aware that he or she was, in fact, performing less consistently when auditory localization was requested, and thus have reflected this awareness in the CR judgments. Either dynamic is clearly more cognitive than perceptual, and either formulation fits the data. In any case, we urge caution in the use of indices with a cognitive component in the study of perceptual processes.

\section{REFERENCES}

Gibson, J. J. Adaptation, after-effect and contrast in the perception of curved lines. Journal of Experimental Psychology, 1933, 16, 1-31.

Hay, J. C., Pick, H. L., JR., \& Ikeda, K. Visual capture produced by prism spectacles. Psychonomic Science, 1965, 2 , 215-216.

Pick, H. L., JR., Warren, D. H., \& Hay, J. C. Sensory conflict in judgments of spatial direction. Perception \& Psychophysics, 1969, 6, 203-206.

WarRen, D. H., \& Cleaves, W. T. Visual-proprioceptive interaction under large amounts of conflict. Journal of Experimental Psychology, 1971, 90, 206-214.

Warken, D. H., Welch, R. B., \& McCarthy, T. J. The role of visual-auditory "compellingness" in the ventriloquism effect: Implications for transitivity among the spatial senses. Perception \& Psychophysics, 1981, 30, 557-564.

Welch, R. B., \& WARREN, D. H. Immediate perceptual response to intersensory discrepancy. Psychological Bulletin, 1980, 88, 638-667.

Youna, P. T. Auditory localization with acoustical transportation of the ears. Journal of Experimental Psychology, 1928, 11, 399-429.

(Manuscript received May 3, 1982;

revision accepted for publication December $29,1982$. 